\title{
SEMANTIC ANNOTATIONS ON HERITAGE MODELS: 2D/3D APPROACHES AND FUTURE RESEARCH CHALLENGES
}

\author{
V. Croce $^{1 *}$, G. Caroti $^{1}$, L. De Luca ${ }^{2}$, A. Piemonte ${ }^{1}$, P. Véron ${ }^{3}$ \\ ${ }^{1}$ Civil and Industrial Engineering Department (DICI), A.S.T.R.O. Laboratory, University of Pisa \\ Largo Lucio Lazzarino 1, 56122 Pisa, Italy - valeria.croce@unifi.it; (gabriella.caroti, andrea.piemonte)@ing.unipi.it \\ ${ }^{2}$ UMR 3495 MAP CNRS/MCC, campus CNRS Joseph-Aiguier, bâtiment US, 31, rue Joseph-Aiguier, 13402 Marseille, France - \\ livio.deluca@map.cnrs.fr \\ ${ }^{3}$ LISPEN EA 7515, Arts et Métiers ParisTech, 13100 Aix-en-Provence, France - philippe.veron@ensam.eu
}

\author{
Commission II, WG II/8
}

KEY WORDS: Cultural Heritage, Semantic annotation, Aïoli, 2D/3D mapping, H-BIM.

\begin{abstract}
:
Research in the field of Cultural Heritage is increasingly moving towards the creation of digital information systems, in which the geometric representation of an artifact is linked to some external information, through meaningful tags. The process of attributing additional and structured information to various elements in a given digital model is customarily identified with the term semantic annotation; the added contextual information is associated, for instance, to analysis and conservation terms. Starting from the existing literature, aim of this work is to discuss how semantic annotations are used, in digital architectural heritage models, to link the geometrical representation of an artefact with knowledge-related information. Most consolidated methods -such as traditional mapping on 2D media, are compared with more recent approaches making the most of 3D representation. Reference is made, in particular, to Heritage-BIM techniques and to collaborative reality-based platforms, such as Aïoli (http://aioli.cloud). Potentialities and limits of the different solutions proposed in literature are critically discussed, also addressing future research challenges in Cultural Heritage application fields.
\end{abstract}

\section{INTRODUCTION}

In recent years, developments in computer graphics have increasingly fostered in virtual environments the use and exploitation of digital models, aiming to replicate real and existing objects.

In the Cultural Heritage domain, the generation of numerical models of heritage objects and historical sites is today a common practice; methods for digitally synthesizing and manipulating heritage content are countless and widely proven (Scopigno et al., 2011).

Beyond the plain and consolidated techniques of sharing and visualization of architectural heritage models, however, the challenges of current research aim at a further achievement, which concerns the generation of digital information systems. Through systems of such kind, the geometrical model can be associated with valuable information relating to documentation, preservation, conservation and restoration activities over the heritage asset.

Digital contents can thus be a meaningful and effective tool, in charge of archeologists, restorers, conservators, engineers and architects, to support the study and analysis of a given architectural object or site.

In pursuing this objective, it is essential to create a link between purely geometric representation (derived from the $3 \mathrm{D}$ model) and semantic representation (associated with external and complementary information about the architectural artifact).

The connection between these two types of representation can be achieved through the insertion of a so-called semantic annotation, understood as a mechanism of association between the graphically represented element and relevant information.
With the review of existing literature on the subject, purpose of this contribution is to illustrate how semantic annotations can link geometry and semantics over digital architectural heritage models, comparing 2D/3D annotation benchmarks.

In Section 2, the definition of semantic annotations in cultural heritage domain is provided and different workflows proposed over time by the research community are concisely analyzed and commented on.

In Section 3, more traditional and consolidated methods of annotation - such as mapping on 2D media - are compared with more recent and innovative approaches based on a 3D representation.

Among the latter, the reference is also to Heritage-Building Information Modeling (H-BIM) techniques and to collaborative reality-based annotation platforms, such as Aïoli (http://aioli.cloud).

Section 4 highlights strengths and weaknesses of the different solutions proposed in literature, even addressing future research challenges in Cultural Heritage application fields.

\section{SEMANTIC ANNOTATION}

The semantic annotation is defined as an operation that associates a graphic element (or part of it) of a digital model with some related semantic information.

As stated by Ponchio et al. (2020), the annotation indicates at the same time the procedure of a) selecting a location/region over a model and b) creating an explicit link between that spatial element and structured, semi-structured or unstructured data.

In case the connection is set with a structured and formalized knowledge, then the annotation is semantic.

* Corresponding author 
The semantic annotation procedure has been widely used in several research fields: in the medical sciences, for the interpretation of anatomical 3D models to support diagnosis and treatment of pathologies (Muller and Unay, 2017; Banerjee et al., 2015); in information sciences and semantic web, to create machine-readable content and semantically tagged documents that can be interpreted, combined and reused by computers (Kalboussi et al., 2015; Slimani, 2013); in industrial manufacturing and supply chains, to assist storage and information exchange during the product life cycle management (Liao et al., 2015).

With the improvement of digital modeling and computer-aided design in the Cultural Heritage domain, this concept has also been specifically extended to architectural objects and historic sites.

The semantic annotation allows to link the geometric representation of the object (quantitative and metric information) to complementary information produced within heritage studies (qualitative information). The latter may concern, for instance, analyses of materials, crack patterns, intervention and recovery measures.

Heterogeneous information, produced during research and analysis on an architectural artifact, can thus be sorted within an intelligible digital representation (De Luca, 2011).

However, the hierarchical organization of this added information is not always the same. For instance, the four levels of semantic enrichment identified by Andrews et al. (2012):

- Tags: keywords or plain terms linked to a given resource;

- Attributes: a characteristic of the resource, defined by a name and an associated value;

- Relations: interlinking of provided resources;

- Ontologies: a specification of a shared conceptualization; can be suitably adapted to the case of digital heritage models.

Tags represent the most trivial form of annotation, being small and clickable notes that can be put in specific positions of the model. Many on-line repositories of 3D models, e.g. Sketchfab (https://sketchfab.com/), 3DHop (http://vcg.isti.cnr.it/3dhop/) and Potree (http://potree.org/), are equipped with this type of annotation tools, to create simple linkable digital content.

Conversely, ontologies represent the most complete form of annotation, as they contain information structured according to a formal naming and a shared classification. Tags, attributes, relations and ontologies allow to spatialize the information, i.e. to directly link it to a $2 \mathrm{D}$ or $3 \mathrm{D}$ representation of the digital model, on different levels of data organization and management.

\section{CLASSIFICATION OF SEMANTIC ANNOTATION PIPELINES}

Starting from the existing literature, scope of this section is to concisely review and classify the different approaches proposed over time for the semantic annotation of digital architectural heritage models (Figure 1). A distinction is made on the basis of: type of annotated media, degree of automation and part/whole relationship.

\subsection{Classification based on type of media}

In Cultural Heritage, the annotation implies a thematic mapping, associating the graphic representation of an asset with knowledge-related information. Depending on the type of support used to execute this thematic mapping, we distinguish:

\section{a) 2D approaches.}

Traditionally, information mapping is a typical tool of 2D media. This approach is based on a bi-dimensional geometric

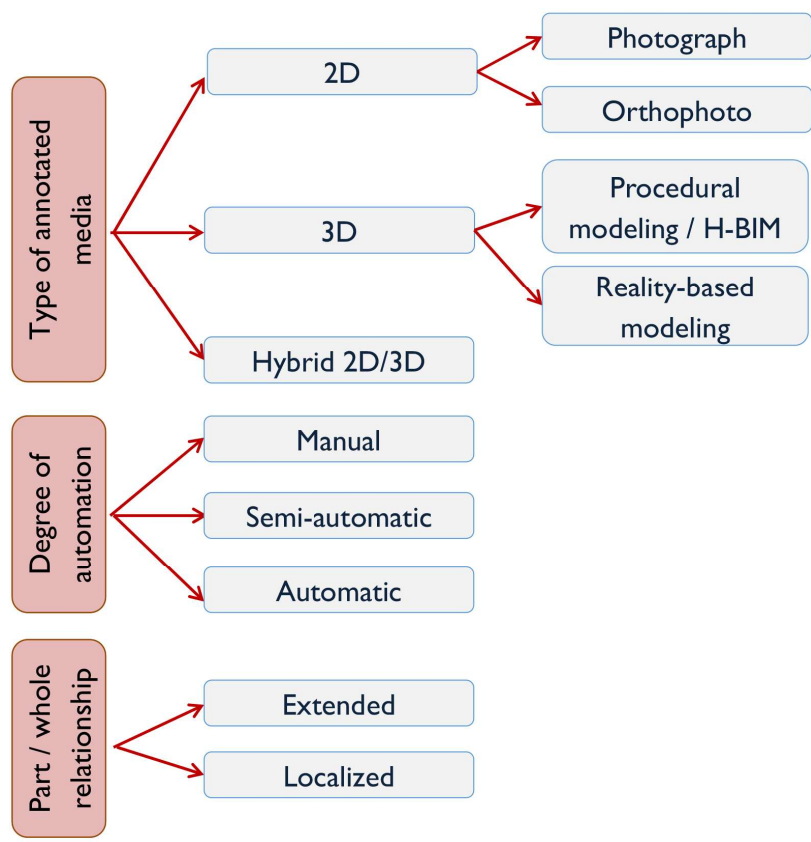

Figure 1. Classification of semantic annotation over digital heritage models

representation: for instance, perspective, for photographs, or orthogonal projection, for orthophotos.

In the conventional approach to degradation or alterations mapping, for example, a certain pattern is applied over a defined area, and its meaning is clarified in a legend. This annotation process is long and time-consuming and requires the execution of countless and diversified graphic drawings. It thus causes not only a large dispersion, but also possible alterations of data.

To overcome some of these limits, the first 2D digital annotation platforms were introduced. They followed the same principle as the Geographic Information Systems (GIS) for land use: the management of multiple layers of information and multiple temporal states over a single 2D media.

A noteworthy result is SICaR, promoted by the Italian Ministry of Culture since 2003 (http://sicar.beniculturali.it/website/), a web platform for the documentation of restoration site activities. The state of conservation and deterioration of monuments is mapped over digital orthophotos (Fabiani et al., 2016).

However, this kind of systems allows a multilayer management on the single orthophoto, but not yet on the whole heritage element.

Although being conceptually referred to a same physical entity an architectural element or parts of it, from the spatial representation point of view the represented 2D data have no direct connection one another. The problem is described in literature as a spatial referencing gap (Messaoudi et al., 2018). This can only be solved by moving to a $3 \mathrm{D}$ representation.

b) 3D approaches.

The semantic annotation procedure is no longer limited to a $2 \mathrm{D}$ support, in fact more recently it has been fruitfully extended to the $3 \mathrm{D}$ media (Figure 2).

It is to say, the annotation is not performed on a two-dimensional projection of the element, but on its real extension representation - in the three dimensions.

These 3D approaches apply both to: reality-based models, derived directly from 3D survey procedures, such as laser scanning and photogrammetry, as well as to conceptually 

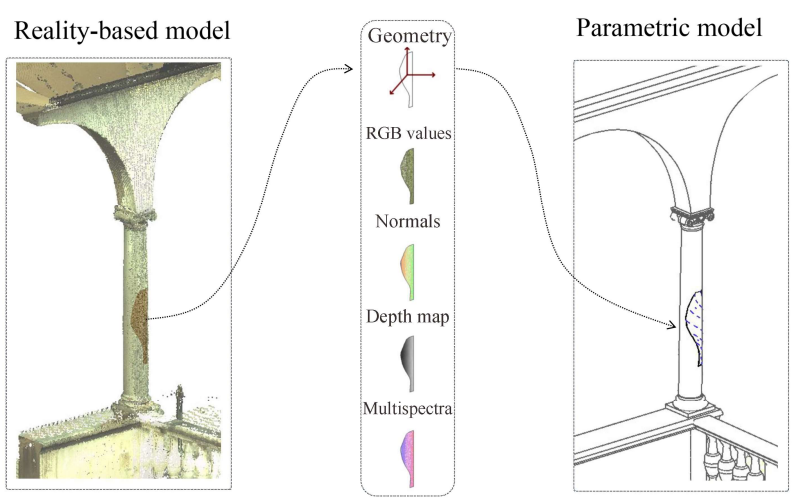

Figure 2. Decay mapping on reality-based and parametric models

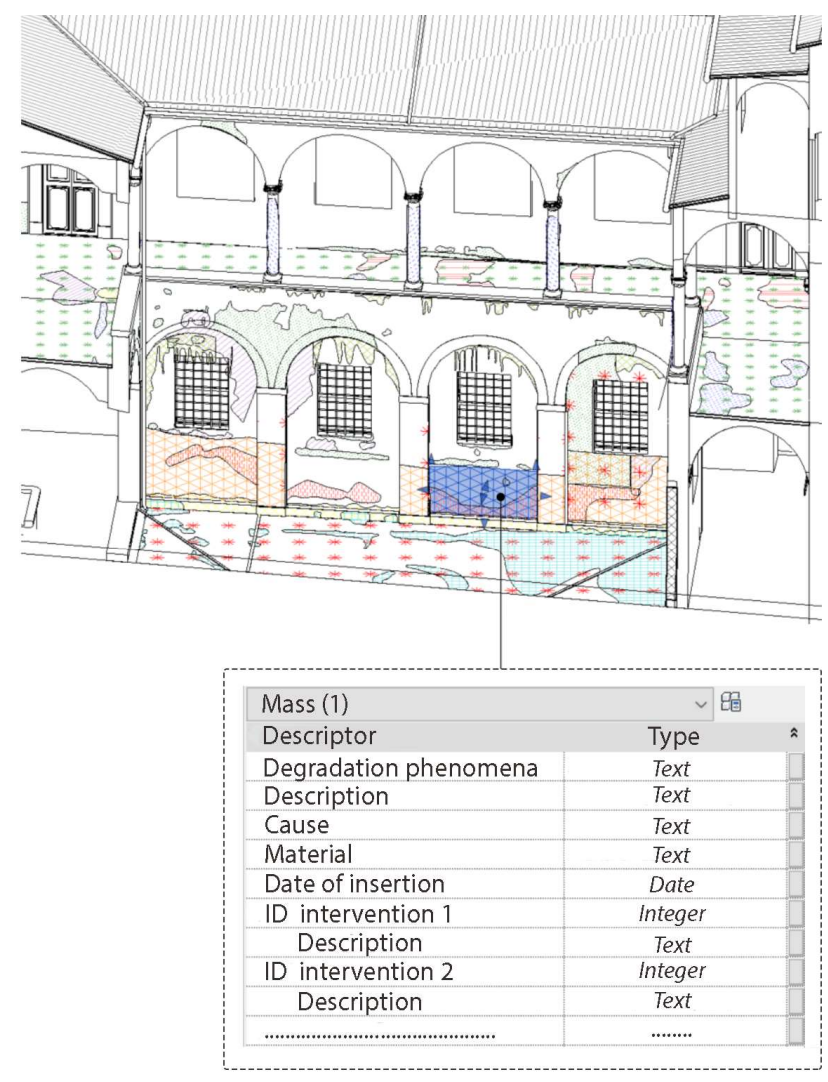

Figure 3. Tags and attributes type annotations within H-BIM platforms

modeled mockups (Bevilacqua et al., 2018; Croce et al., 2019ab).

As for reality based-models, annotations consist in selecting a functional subset over the mesh or the point cloud.

Few web platforms -the already mentioned Sketchfab, Potree and 3DHop or the applications described in Soler et al. (2013) and Attene et al. (2009) offer simple tools to insert annotations (tags) of this type, where the user selects a part of the model and then adds simple tags. That allows high level of user-friendliness, which guarantees their use even by less-experienced operators; but this undeniable advantage is also the limit of such platforms. For that reason, several modern studies in the field of Cultural Heritage focus on the creation of annotation platforms aiming at a more semantic-aware 3D representation.

Following there is a concise list of existing platforms, stemming from these up to date studies.
The 3D Semantic Annotation System (3DSA) presented by Yu et al. (2013) supports rule-based reasoning across generated annotations to catalogue heritage artifacts. Scalas et al. (2020), in the context of the GRAVITATE project, build a 3D semantic annotation system aimed at the virtual assembly of fragmented or broken heritage artifacts.

More thoroughly in the architectural heritage domain, Apollonio et al. (2018) propose a web-based information system to support the restoration of the Neptune's Fountain in Bologna. The 3D model of the heritage object is at the core of the whole system, becoming the pivotal element where to store and semantically organize new and previous restoration data.

With the same principle, the software tool CulTO (Garozzo et al., 2017) is developed to record data within $3 \mathrm{D}$ models of religious historical buildings. The semantic annotation is conceived to enable automatic indexing, classification and correct archiving of texts and iconographic resources of the concerned buildings.

On the other hand, thanks to the spread of Building Information Modeling (BIM) techniques applied to heritage structures (Bruno et al., 2018; López et al., 2018), another type of annotation procedure can be performed, on parametric models. These models display the conceptual shapes of existing heritage buildings, reconstructed from initial survey data via a reverse engineering process, and can be enriched with non-geometric information (Brumana et al., 2020).

H-BIM systems enable to easily perform tag and attribute type annotations (Figure 3), associated to an entire parametric component (a wall, a slab, a column). Supplementary files, such as images, CAD drawings or ASCII files, can be associated with the element.

Obviously, procedures asking for the insertion of annotations that do not concern the parametric element in its entirety, but only a part of it, become more and more complex.

This is the instance that occurs, for example, for the mapping of degradation and decay of materials or crack patterns. Here, the annotations are inserted by exploiting the creation of so-called adaptive components, which are regions suitably associated with either flat or curved surfaces of each parametric element (Chiabrando et al., 2017; Lo Turco et al., 2017).

Malinverni et al. (2019), for instance, leverage on adaptive components to build a thematic map of degradation, with attached legend. Each annotation is categorized with a proper ID code assignment, to ease the accomplishment of specific queries within the model. Simeone et al. (2019) apply this procedure to detect transformations and reused elements within the northern wall of the Oratory of San Saba, in Rome.

Pocobelli et al. (2018) perform annotations in H-BIM environment in order to construct a weathering forecasting model, showing the spatial distribution of moisture along surface walls.

Bacci et al. (2019), with a similar procedure, investigate the logical structuring of annotations, by associating the adaptive components to multicategory tags, related to degradation level, material and/or conservation interventions.

\section{c) Hybrid $2 D / 3 D$ approaches.}

Hybrid approaches start from the assumption that it is much easier to select the annotation on a 2D media rather than on the 3D model. They thus allow the performance of an annotation over images or orthographic drawings and then exploit projective relationships to automatically transfer the annotation from the 2D model to the 3D media.

The NUBES web-based platform (Stefani et al., 2014) represents a first attempt towards the development of this methodology. It relies on the extraction of textures from the $3 \mathrm{D}$ model, by means 


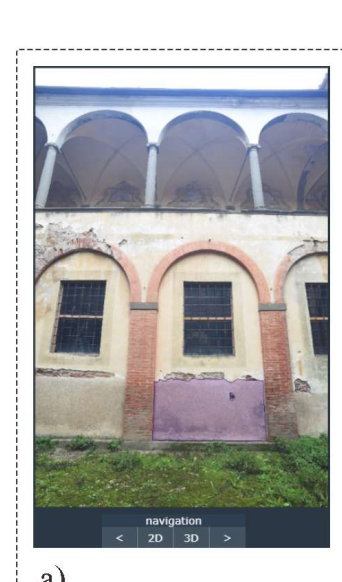

a)
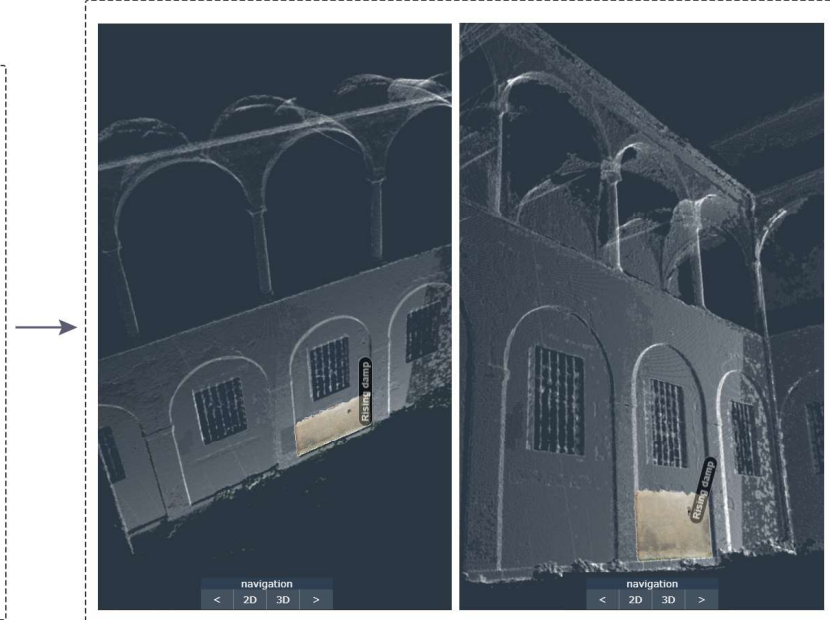

b)

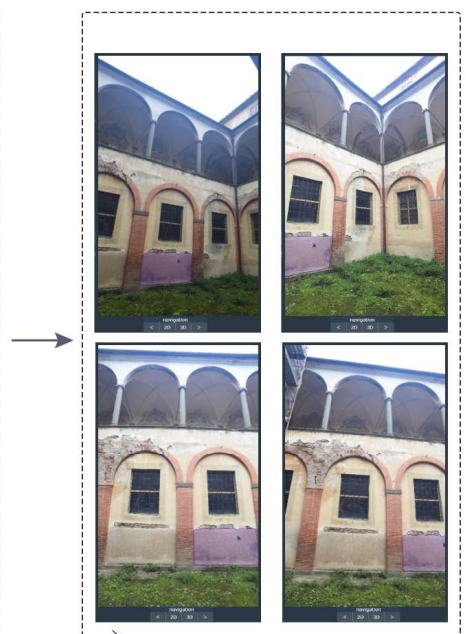

c)

Figure 4. Processing steps via Aïoli: (a) selection of the annotation on the first image, (b) detection of 3D points corresponding to the selection and (c) propagation of the annotation on the other images of the set

of a suitable cubic, planar or spherical projection. The unfolded textures are annotated with information on stone degradation, dating and material; then, they are projected back and displayed onto the 3D model, through a proper reprojection matrix.

More innovative system, working on a set of images calibrated and spatially oriented on the $3 \mathrm{D}$ model, have been recently envisaged.

The Aioli collaborative platform, developed at the MAP laboratory of the CNRS Marseille, is built upon this concept (Manuel et al., 2016, 2014).

Aioli involves the creation of a model by photogrammetric process (point-based 3D model). For each image, Aïoli stores a file that binds pixels of the image with associated $3 \mathrm{D}$ points. The operator picks the image better displaying the region to be annotated and traces the selection (by using drawing tools on the 2D support).

By recreating the $2 \mathrm{D} / 3 \mathrm{D}$ correspondences, the annotated region is transferred to the 3D model and then projected back to all other images where it is visible.

In doing so, the semantic annotation is not only transferred from the initial image to the $3 \mathrm{D}$ representation, but it is also propagated to all other photographs where it is shown (Figures 4-5).

A continuous projective relationship is created, moving in two directions: from $2 \mathrm{D}$ to $3 \mathrm{D}$ and from $3 \mathrm{D}$ to $2 \mathrm{D}$ (Figure 6).

Annotated, semantically enriched models can be shared between users via cloud computing services, which is why the platform is collaborative. A study on the use of Aioli aimed at annotating degradation phenomena over sculptural heritage artifacts has been previously illustrated in Roussel et al. (2019).

\subsection{Classification based on degree of automation}

Semantic annotations are classified as:
a) Manual;
b) Semi-automatic,
c) Automatic,

according to their degree of automation. Although the classification usually proposed in literature refers to $2 \mathrm{D}$ media (Manuel et al., 2016), its extension in case of 3D models is quite natural.

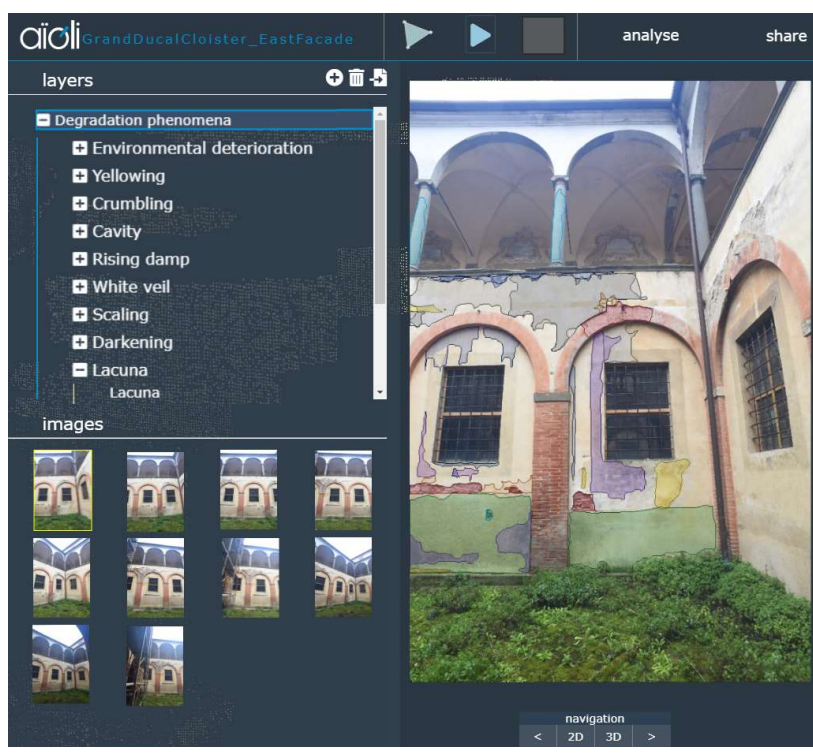

Figure 5. Insertion of different layers of degradation phenomena on Aïoli

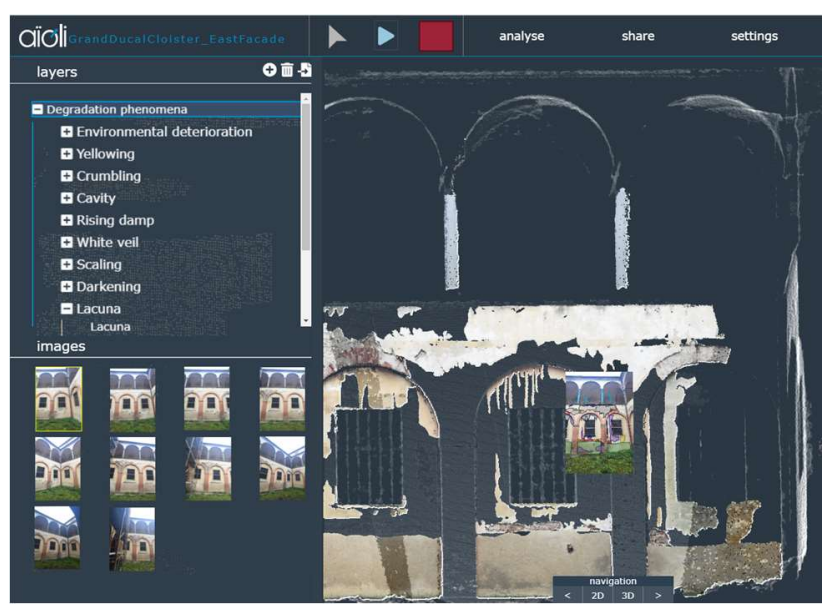

Figure 6. Projection of the annotations in $2 \mathrm{D} / 3 \mathrm{D}$ 
In case of manual annotation, the region of interest is manually indicated by the end-user, through free-form or polygonal selection tools.

This is the most widespread method and allows a greater insight and control by the user, who associates the semantic tag himself. Since driven by the user in every step, on the one hand this approach can be very accurate, on the other hand it is timeconsuming and strongly dependent on the visual judgement and on the skill of the user himself (Ponchio et al., 2020).

For this reason, new approaches to annotation insertion have been developed more recently, based on the automatic or semiautomatic identification of meaningful attributes over the model. On reality-based models, regions of interest can be detected based on some extracted features.

This process involves the phases of segmentation, features extraction, features selection and classification.

Existing 2D/3D segmentation and classification techniques applied to digital heritage models have been comprehensively illustrated in Grilli et al. (2017) and Grilli and Remondino (2019). Also concerning BIM platforms, many solutions envisaging the automatic recognition and parameterization of some plain elements, such as walls and floors, have been proposed (Macher et al., 2017; Tang et al., 2010).

However, these strategies apply for trivial components and only if the element to detect is the whole parametric element (e.g. a single wall), not some parts of it (e.g. to assess a localized alteration phenomenon on the wall).

Automatic or semi-automatic annotation of degradation phenomena, for instance, is an attainable target in the case of reality-based models but not in the case of parametric models.

\subsection{Classification based on part/whole relationship}

As emerged from Sections 3.1 and 3.2, a third methodology of classification can be proposed aiming to allow a more flexible association of the semantic annotations.

In that methodology, based on the part/whole relationship, the semantic annotation can thus concern, in turn, restricted, localized areas, the whole element or even the entire model.

In H-BIM platforms, whatever conceived, semantic annotations on parametric components considered in their entirety are very easy; however, introducing suitable parametric adaptive components, it is also possible to extend this capability to localized elements.

This feature is an essential requirement for information systems focused on the restoration and conservation of cultural heritage, where also localized areas need to be annotated, for example, when affected by localized damage or degradation phenomena.

\section{CRITICAL DISCUSSION}

2D and 3D digital supports can both be semantically enriched through an annotation process. However, the shift to a 3D media allows a complete and clear representation of the heritage object. The semantic annotations can be inserted in 3D models using two distinct representations methods.

In fact, while H-BIM platforms are based on adaptive components created within a parametric representation, other up to date collaborative platforms, like Aïoli, rely on reality-based acquisitions and on the creation of an informative continuum between 2D images and 3D models.

Both methods offer noticeable advantages in comparison with conventional 2D mapping:

1. Multilayer management intended as the capability to handle multiple layers of information over the same representation.
Knowledge on degradation, crack patterns, material and type of intervention can be linked to the same semantic 3D model, virtually tying up annotations to its parts.

In this way, data overlapping is permitted; consequently, data do not have to be duplicated, as is the case of traditional cartographic mapping.

2. Multitemporal management intended as the possibility to represent different temporal states over the same representation (e.g. temporal evolution of degradation mechanisms or crack patterns).

3. Knowledge-based management. If the semantic information within the model is inserted according to formalized logic, like in case of ontologies, the information management mechanism can be univocally determined, thus providing sound bases for its extensive acceptance and recognition.

This means that different experts can insert the same annotation in a univocal manner.

4. Data interpolation. Cross-check of data and information retrieval through queries could be very beneficial when correlation elements are explored (e.g. between materials and state of degradation, or between state of degradation, crack pattern and outcomes of past interventions, also in view of the assessment of their evolution).

As already stressed, annotations performed on H-BIM platforms are straightforward if the information is to be connected to entire parametric elements, rather than to parts of it, but in case of annotation of degrading parts, it is necessary to introduce more refined annotation techniques, based on the suitable adaptive components expedient.

Moreover, the export of data related to adaptive components, for instance aiming to share the annotations on the web, is still a long and tedious process.

Sounder support of interoperability scenarios remains thus requires further studies.

Nevertheless, BIM platforms allow annotations of the type tag and attribute, thus enabling plain computation operations (statistical analyses), devoted, for example, to estimate the actual extension of the annotation to support restoration activities (Lo Turco et al., 2017).

From the above considerations, it clearly emerges that realitybased collaborative platforms including all four aspects of annotation listed by Andrews et al. (2012) seem much more effective, allowing higher level of semantic enrichment.

Among them, particular attention is devoted to already re-called Aïoli, an open source platform specifically designed for cloud computing services.

Aïoli enhances data transfer between 2D/3D media, ensuring interoperability, data exchange and propagation of information within the as wide as possible audience of archaeologists, restorers, conservators, architects and engineers.

With this principle, an annotation can also be updated over time, for example by calibrating and orienting a new set of photos or updating archive information over the considered model.

In addition, the exploitation of photogrammetric models allows to store radiometric (color-related) information, too.

However, the precision of annotations performed over point clouds or mesh models strongly depends on the accuracy of the original model (in terms of density and precision of points for the point cloud and number and shape of triangular and polygonal elements in a mesh).

Finally, both methods are mostly based on manual selection of the annotations. 


\section{CONCLUSION AND FUTURE RESEARCH CHALLENGES}

In this contribution, the advantages of semantic annotations over $3 \mathrm{D}$ or hybrid $2 \mathrm{D} / 3 \mathrm{D}$ media are examined, in comparison with the conventional 2D mapping. Existing literature on the subject is concisely presented.

Reference is made to most recent Heritage-BIM techniques and to collaborative reality-based platforms, such as Aïoli.

These two methodologies allow at different levels: multilayer management, multitemporal management, knowledge-based management and data interpolation, but several open issues need deeper investigations, which are the subject of studies actually in progress.

The most promising research lines concern the improvement of the following relevant topics:

\section{a) Construction of a formalized knowledge base}

Heterogeneous information should be organized within the model by setting up a unique data entry process, aiming to form a common basis to be recognized and shared by the different experts working on the heritage asset.

By formalizing the knowledge related to a specific domain, different data can be collected and structured based on a machinereadable rationale and on controlled specific glossary and vocabulary.

It is a matter of semantically enriching the representation through ontologies (the fourth level of semantic annotation).

The above-mentioned work by Messaoudi et al. (2018) implements a data model based on a domain ontology for the reality-based 3D annotation of heritage building conservation state. It aims in particular at connecting the threefold facets of the digital heritage model concerning spatial, morphological and semantic features.

\section{b) Annotation transfer between different representation systems}

As shown above, in Section 3.1, semantic annotations can be performed over different media; for this reason, the output of a same semantic annotations may vary based on the model resolution and on the type of representation (e.g. different number of triangles or polygonal surfaces of a mesh, different point clouds density).

A research challenge thus concerns the development of a system of input and retrieval of information, to be:

- Independent of the type of representation (software used, accuracy and scale of the 2D or 3D representation and so on); - Insensitive on modifications of the geometry due to refinements of the model, e.g. availability of additional data, combination of different representation techniques (point clouds, orthophotos, mesh, parametric models).

This task is raised in a recent study by Scalas et al. (2020), that addresses this issue as annotation persistence and develops a first pipeline for the annotation transfer between mesh models at different resolution.

The achievement of the annotation persistence, leveraging annotation transfer between multiple representations, would ensure continuous update and refinement of semantic annotations over time, regardless of the type of model used.

\section{c) More automated semantic annotation procedures}

The semantic annotation procedure, also in view of the annotation persistence, could be greatly expedited with the introduction of automatic or semi-automatic techniques for the detection of regions of interest.

These techniques leverage the segmentation of the model based on similar characteristics and the subsequent semantic classification (labeling) of the elements.

Their application would lead to a significative reduction of the human involvement in the brute annotation phase, lessening the space of arbitrary or subjective choices.

This results particularly promising for detection and labeling, over 3D models, of recurrent and repeated typological elements, such as walls and slabs, columns, capitals, vaults, mullioned windows, rose windows or even more complex elements, whatever the scope and the extension of the annotation.

These possible future research developments aim to streamline the annotation procedure and to foster the exchange of research results on heritage architectural assets, even with access privileges, depending on the user typology, on a platform independent basis, to improve the archival and retrieval of information and digital documentation, as well as their dissemination.

\section{ACKNOWLEDGEMENTS}

The presented work is the first result of a collaboration between the Department of Civil and Environmental Engineering of the University of Florence, the Department of Civil and Industrial Engineering of the University of Pisa (Italy), the MAP laboratory of the Centre National de la Recherche Scientifique CNRS Marseille and the LISPEN Laboratory in Aix-en-Provence (France), and it is carried out in the framework of a Ph.D. cotutelle agreement. It is partially funded by the VINCI2019 Project, promoted by the Université Franco-Italienne.

\section{REFERENCES}

Andrews, P., Zaihrayeu, I., Pane, J., 2012. A classification of semantic annotation systems. Semantic Web 3, 223-248.

Apollonio, F.I., Basilissi, V., Callieri, M., Dellepiane, M., Gaiani, M., Ponchio, F., Rizzo, F., Rubino, A.R., Scopigno, R., Sobra', G., 2018. A 3D-centered information system for the documentation of a complex restoration intervention. Journal of Cultural Heritage 29, 89-99.

Attene, M., Robbiano, F., Spagnuolo, M., Falcidieno, B., 2009. Characterization of 3D shape parts for semantic annotation. Computer-Aided Design 41, 756-763.

Bacci, G., Bertolini, F., Bevilacqua, M.G., Caroti, G., MartínezEspejo Zaragoza, I., Martino, M., Piemonte, A., 2019. H-BIM methodologies for the architectural restoration: the case of the exchurch of San Quirico all'Olivo in Lucca, Tuscany. Int. Arch. Photogramm. Remote Sens. Spatial Inf. Sci. XLII-2/W11, 121126.

Banerjee, I., Agibetov, A., Catalano, C.E., Patane, G., Spagnuolo, M., 2015. Semantic Annotation of Patient-Specific 3D Anatomical Models, in: 2015 International Conference on Cyberworlds $(\mathrm{CW})$. Presented at the 2015 International Conference on Cyberworlds (CW), IEEE, Visby, Sweden, pp.

Bevilacqua, M.G., Caroti, G., Piemonte, A., Terranova, A.A., 2018. Digital Technology and Mechatronic Systems for the Architectural 3D Metric Survey, in: Ottaviano, E., Pelliccio, A., Gattulli, V. (Eds.), Mechatronics for Cultural Heritage and Civil Engineering, Intelligent Systems, Control and Automation: Science and Engineering. Springer International Publishing, Cham, 161-180. 
Brumana, R., Oreni, D., Barazzetti, L., Cuca, B., Previtali, M., Banfi, F., 2020. Survey and Scan to BIM Model for the Knowledge of Built Heritage and the Management of Conservation Activities, in: Daniotti, B., Gianinetto, M., Della Torre, S. (Eds.), Digital Transformation of the Design, Construction and Management Processes of the Built Environment. Springer International Publishing, Cham, 391400.

Bruno, S., De Fino, M., Fatiguso, F., 2018. Historic Building Information Modelling: performance assessment for diagnosisaided information modelling and management. Automation in Construction 86, 256-276.

Chiabrando, F., Lo Turco, M., Rinaudo, F., 2017. Modeling the decay in an HBIM starting from 3D point clouds. A followed approach for Cultural Heritage knowledge. Int. Arch. Photogramm. Remote Sens. Spatial Inf. Sci. XLII-2/W5, 605612.

Croce, V., Caroti, G., Piemonte, A., Bevilacqua, M.G., 2019. Geomatics for Cultural Heritage conservation: integrated survey and 3D modeling. In: Proc. of the IMEKO TC4 International Conference on Metrology for Archaeology and Cultural Heritage, MetroArchaeo, Dec. 4-6, 2019, Florence, Italy, 271276.

Croce, V., Caroti, G., Piemonte, A., 2019. Assessment of earthquake-induced damage level on buildings: analysis of two different survey methods for a case study, Int. Arch. Photogramm. Remote Sens. Spatial Inf. Sci., XLII-2/W15, 351358 ,

De Luca, L., 2011. Methods, formalisms and tools for the semantic-based surveying and representation of architectural heritage. Appl Geomat. 6, 115-139.

Fabiani, F., Grilli, R., Musetti, V., 2016. Verso nuove modalità di gestione e presentazione della documentazione di restauro: SICaR web la piattaforma in rete del Ministero dei Beni e delle Attività Culturali e del Turismo. Bollettino Ingegneri, Collegio Ingegneri della Toscana 3-13.

Garozzo, R., Murabito, F., Santagati, C., Pino, C., Spampinato, C., 2017. CulTO: an ontology-based annotation tool for data curation in Cultural Heritage. Int. Arch. Photogramm. Remote Sens. Spatial Inf. Sci. XLII-2/W5, 267-274.

Grilli, E., Menna, F., Remondino, F., 2017. A review of point clouds segmentation and classification algorithms. Int. Arch. Photogramm. Remote Sens. Spatial Inf. Sci. XLII-2/W3, 339344.

Grilli, E., Remondino, F., 2019. Classification of 3D Digital Heritage. Remote Sensing 11, 847.

Kalboussi, A., Omheni, N., Mazhoud, O., Kacem, A.H., 2015. How to Organize the Annotation Systems in Human-Computer Environment: Study, Classification and Observations, in: Abascal, J., Barbosa, S., Fetter, M., Gross, T., Palanque, P., Winckler, M. (Eds.), Human-Computer Interaction INTERACT 2015, Lecture Notes in Computer Science. Springer International Publishing, Cham, pp. 115-133.
Liao, Y., Lezoche, M., Panetto, H., Boudjlida, N., Loures, E.R., 2015. Semantic annotation for knowledge explicitation in a product lifecycle management context: A survey. Computers in Industry 71, 24-34.

Lo Turco, M., Mattone, M., Rinaudo, F., 2017. Metric survey and BIM technologies to record decay conditions. Int. Arch. Photogramm. Remote Sens. Spatial Inf. Sci. XLII-5/W1, 261268.

López, F., Lerones, P., Llamas, J., Gómez-García-Bermejo, J., Zalama, E., 2018. A Review of Heritage Building Information Modeling (H-BIM). MTI 2, 21.

Macher, H., Landes, T., Grussenmeyer, P., 2017. From Point Clouds to Building Information Models: 3D Semi-Automatic Reconstruction of Indoors of Existing Buildings. Applied Sciences 7, 1030.

Malinverni, E.S., Mariano, F., Di Stefano, F., Petetta, L., Onori, F., 2019. Modeling in HBIM to document materials decay by a thematic mapping to manage the Cultural Heriage: the case of Chiesa della Pietà in Fermo. Int. Arch. Photogramm. Remote Sens. Spatial Inf. Sci. XLII-2/W11, 777-784.

Manuel, A., Véron, P., De Luca, L., 2016. 2D/3D Semantic Annotation of Spatialized Images for the Documentation and Analysis of Cultural Heritage. Eurographics Workshop on Graphics and Cultural Heritage, 101-104.

Messaoudi, T., Véron, P., Halin, G., De Luca, L., 2018. An ontological model for the reality-based 3D annotation of heritage building conservation state. Journal of Cultural Heritage 29, $100-112$.

Muller, H., Unay, D., 2017. Retrieval From and Understanding of Large-Scale Multi-modal Medical Datasets: A Review. IEEE Trans. Multimedia 19, 2093-2104.

Pocobelli, D.P., Boehm, J., Bryan, P., Still, J., Grau-Bové, J., 2018. Building Information Models for monitoring and simulation data in heritage buildings. Int. Arch. Photogramm. Remote Sens. Spatial Inf. Sci. XLII-2, 909-916.

Ponchio, F., Callieri, M., Dellepiane, M., Scopigno, R., 2020. Effective Annotations Over 3D Models. Computer Graphics Forum 39, 89-105.

Roussel, R., Bagnéris, M., De Luca, L., Bomblet, P., 2019. A digital diagnosis for the "autumn" statue (Marseille, France): photogrammetry, digital carthography and construction of a thesaurus. Int. Arch. Photogramm. Remote Sens. Spatial Inf. Sci. XLII-2/W15, 1039-1046.

Scalas, A., Mortara, M., Spagnuolo, M., 2020. A pipeline for the preparation of artefacts that provides annotations persistence. Journal of Cultural Heritage 41, 113-124.

Scopigno, R., Callieri, M., Cignoni, P., Corsini, M., Dellepiane, M., Ponchio, F., Ranzuglia, G., 2011. 3D Models for Cultural Heritage: Beyond Plain Visualization. Computer 44, 48-55.

Simeone, D., Cursi, S., Acierno, M., 2019. BIM semanticenrichment for built heritage representation. Automation in Construction 97, 122-137.

Slimani, T., 2013. Semantic Annotation: The Mainstay of Semantic Web. IJCATR 2, 763-770. 
Soler, F., Torres, J.C., León, A.J., Luzón, M.V., 2013. Design of cultural heritage information systems based on information layers. J. Comput. Cult. Herit. 6, 1-17.

Stefani, C., Brunetaud, X., Janvier-Badosa, S., Beck, K., De Luca, L., Al-Mukhtar, M., 2014. Developing a toolkit for mapping and displaying stone alteration on a web-based documentation platform. Journal of Cultural Heritage 15, 1-9.

Tang, P., Huber, D., Akinci, B., Lipman, R., Lytle, A., 2010. Automatic reconstruction of as-built building information models from laser-scanned point clouds: A review of related techniques. Automation in Construction 19, 829-843.

Wang, Z., Shi, W., Akoglu, K., Kotoula, E., Yang, Y., Rushmeier, H., 2018. CHER-Ob: A Tool for Shared Analysis and Video Dissemination. J. Comput. Cult. Herit. 11, 1-22.

Yu, C.-H., Groza, T., Hunter, J., 2013. Reasoning on CrowdSourced Semantic Annotations to Facilitate Cataloguing of 3D Artefacts in the Cultural Heritage Domain, in: Salinesi, C., Norrie, M.C., Pastor, Ó. (Eds.), Advanced Information Systems Engineering, Lecture Notes in Computer Science. Springer Berlin Heidelberg, Berlin, Heidelberg, pp. 228-243. 